\title{
On a Nearest-Neighbour Problem in Minkowski and Power Metrics
}

\author{
M.L. Gavrilova \\ Dept of Comp. Science, University of Calgary \\ Calgary, AB, Canada, T2N1N4 \\ marina@cpsc.ucalgary.ca
}

\begin{abstract}
The paper presents an efficient algorithm for solving the nearestneighbor problem in the plane, based on generalized Voronoi diagram construction. The input for the problem is the set of circular sites $S$ with varying radii, the query point $p$ and the metric (Minkowski or power) according to which the site neighboring the query point, is to be reported. The IDG/NNM software was developed for an experimental study of the problem. The experimental results demonstrate that the Voronoi diagram method outperforms the $k-d$ tree method for all tested input site configurations. The similarity between the nearest-neighbor relationship in the Minkowski and power metrics was also established.
\end{abstract}

\section{Introduction}

The Voronoi diagram is often used as a convenient tool for solving scientific problems in computer modeling of physical phenomena. These include structure analysis of unordered systems (liquids, solutions, polymers) [12], stress analysis and simulation of granular systems (ice flow, silo models) [9], and space structures in complex molecular and biological systems 1117.

There are some challenges arising while investigating such problems. Existing software and algorithms are not customized to efficiently solve a variety of application problems. A particular problem addressed in this paper is the finding of a nearest-neighbor in a system of poly-sized circular objects 14. The application of the ordinary point site Voronoi diagram to perform the nearest-neighbor query in 2D is straightforward [1]. The algorithm takes $O(n)$ space, $O(n \log n)$ preprocessing time, and the worst-case running time of $O(n)$. The same idea has been extended to higher dimensions [3], applied to solve the point location problem among the convex sites [16!10], and used to solve the nearest-neighbor problem for dynamic Voronoi diagram [4. The generalized VD in Laguerre geometry was successfully used to solve the collision optimization problem in a system of moving particles [9. The properties of the generalized weighted Voronoi diagrams that enable the use of this data structure for nearest-neighbor detection were thoroughly investigated in [8]. However, there has not been a study that compares the various generalized Voronoi diagrams with respect to solving the nearest-neighbor problem.

Thus, this paper presents a study of the generalized Voronoi diagram approach

V.N. Alexandrov et al. (Eds.): ICCS 2001, LNCS 2073, pp. 663672 2001.

(C) Springer-Verlag Berlin Heidelberg 2001 
for finding the nearest-neighbor for a set of non-intersecting circles. Application domains for this problem can be found in computer graphics, GIS, computer modeling and computer simulation [14. The developed method can also be applied to problems from statistics and information retrieval fields. The data structures studied are the generalized weighted Voronoi diagram (VD) under the Manhattan, supremum and power metrics. The main result is an efficient and robust algorithm for the nearest-neighbor computation in Manhattan, supremum and power metrics.

The performance of the VD based method was compared against the CORE library implementation of the $k-d$ tree method [2] that was modified to handle weighted sites under the supremum metric. The experimental results show significantly better performance for the generalized VD based method, including large (10,000 sites) data sets with various topologies. It is also worth noting that a similar technique can be applied to solve a variety of problems, such as all nearest-neighbors, point location and range queries.

\section{Problem Definition}

Consider a set of circular sites $S$ in the plane. Define a nearest-neighbor relation between the query point $x$ and a site $P$ as follows. The point $x \in R^{2}$ is the nearest neighbor of $P \in S$ iff $d(x, P) \leq \min _{Q \neq P} d(x, Q), Q \in S$. The distance $d(x, P)$ between a point $x\left(x_{1}, x_{2}\right)$ and a circle $P=\left\{p, r_{p}\right\}$ with the center at $p\left(p_{1}, p_{2}\right)$ and radius $r_{p}$ can be computed as

$$
d(x, P)=d(x, p)-r_{p}=\left|x_{1}-p_{1}\right|+\left|x_{2}-p_{2}\right|-r_{p}
$$

in the Manhattan $\left(L_{1}\right)$ metric, and as

$$
d(x, P)=d(x, p)-r_{p}=\max \left(\left|x_{1}-p_{1}\right|,\left|x_{2}-p_{2}\right|\right)-r_{p}
$$

in the supremum $\left(L_{\infty}\right)$ metric. In Laguerre geometry (under the power distance function) it is computed according to the formula:

$$
d(x, P)=d(x, p)^{2}-r_{p}^{2}=\left(x_{1}-p_{1}\right)^{2}+\left(x_{2}-p_{2}\right)^{2}-r_{p}^{2} .
$$

We propose to use the generalized Voronoi diagram as a tool to solve the problem. The generalized Voronoi diagram of a set of circles $S$ in the plane is a set of generalized Voronoi regions $G \operatorname{Vor}(P)$, where

$$
G \operatorname{Vor}(P)=\{x \mid d(x, P) \leq d(x, Q), \forall Q \in S-\{P\}\}
$$

and $d(x, P)$ is the distance function between a point $x$ and a circle $P$ 14. The distance function $d(x, P)$ is defined according to the metric employed. The example of the generalized Voronoi diagram in supremum metric of 1000 sites can be found in Fig. 1. A generalized Delaunay triangulation (DT) is the dual of a generalized Voronoi diagram obtained by joining all pairs of sites whose generalized Voronoi regions share a common edge. The nearest-neighbor property 


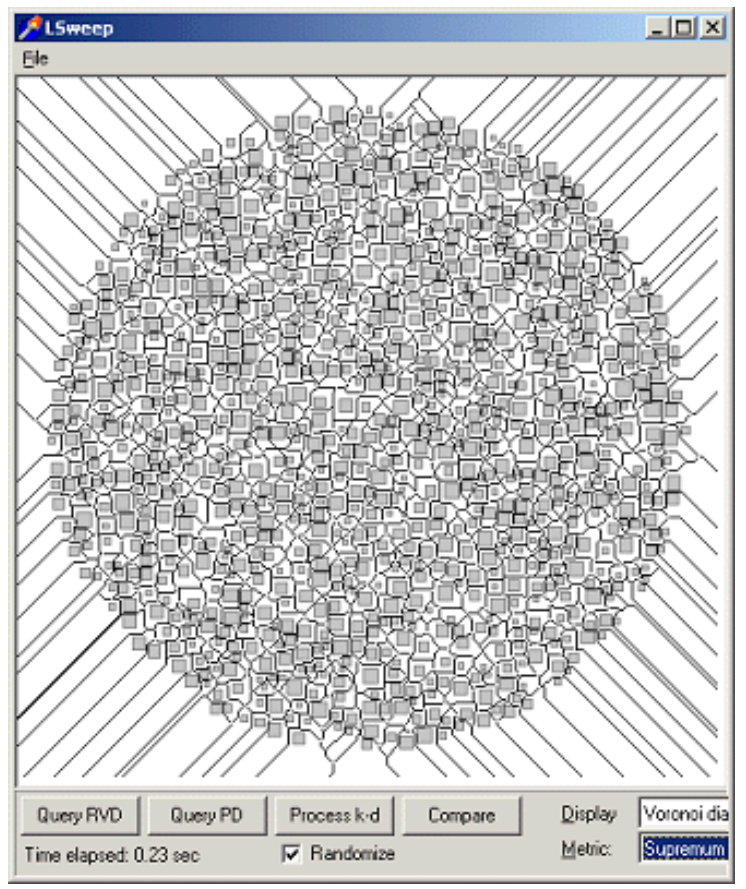

Fig. 1. Supremum weighted VD for 1000 randomly distributed sites generated using the Initial Distribution Generator (IDG) module.

for VD in various metrics, including Manhattan, supremum and power, was established in [8. This property allows solving the nearest-neighbor problem by constructing the generalized Voronoi diagram or the Delaunay triangulation. The method is presented in the following section.

\section{The Nearest-Neighbor Search Algorithm}

The following outlines the nearest-neighbor search algorithm based on the generalized DT construction. The input for the algorithm is a set of circular sites. The approach is based on the simple edge-walk technique that starts with a random location in the Delaunay triangulation:

1. (Initialization) Build the weighted generalized Delaunay triangulation (using, for example, a flip-based incremental construction technique).

2. Find a site $P$ neighboring the query point $x$.

a) Randomly select a site $P_{0}$ as a starting site for the search (call it the active site). 
b) Randomly select an edge adjacent to the active site in the Delaunay triangulation and set its value to $e_{\text {prev }}=e_{\text {curr }}=e_{\text {new }}$

c) Perform a counter-clock wise walk alone the DT edges adjacent to the active site. Select the first edge such that $x$ is located to the left of the straight line passing through this edge, by performing the CCW orientation test.

d) Update $e_{\text {prev }}=e_{\text {curr }}, e_{\text {curr }}=e_{\text {new }}$. Set $e_{\text {new }}$ as the newly found edge.

e) If edges $e_{\text {prev }}, e_{c u r r}, e_{n e w}$ do not form a triangle enclosing the query point $x$, set the endpoint of $e_{\text {new }}$ edge to be the new active site. GOTO 2(c).

3. Report the closest of the vertices of the triangle formed by the edges $e_{\text {prev }}, e_{c u r r}, e_{\text {new }}$ as the nearest-neighbor to the query point $x$.

The preprocessing step is the worst-case optimal $O(n \log n)$, the worst-case number of edges visited during the Step 2 is $O(n)$ (since we never consider a visited edge twice) and the space complexity is $O(n)$.

Note 1: The incremental flipping algorithm description can be found in 5].

Note 2: The algorithm is applicable for solving the point location problem and the range search problem in the presence of specific constraints. Thus, the presented algorithm locates the Voronoi region containing the query point and the generator of this Voronoi region is reported as the nearest neighbor.

Note 3: The Voronoi diagram does not depends on the sizes or distributions of the circles, with the exception of close to degenerate cases, that also require special treatment in the cell-based or $k-d$ tree methods [2].

\section{IDG/NNM Software}

The algorithm outlined above was implemented in the object-oriented Borland Delphi environment. The experiments were conducted on a Pentium II 350 computer with 128 MB RAM. The program consists of two modules. The first module, the Initial Distribution Generator (IDG), is used to create various configurations for the input sites. IDG can generate a new distribution by importing the distribution from a text file, where the coordinates of the centers and radii of circles are specified. IDG can also automatically generate various distributions, such as uniform distribution of sites in the interior of a square, uniform distribution of sites in the interior of a circle, cross, ring, degenerate grid and degenerate circle (see Fig. 21). The parameters of the distribution, including the number of circles, the distribution of their radii, the size of the area, and the type of the distribution must be specified as well. The second module, the Nearest-Neighbor Monitor (NNM), is the program that constructs the additively weighted supremum $\mathrm{VD}$, the power diagram and the $k-d$ tree in supremum metric for the 

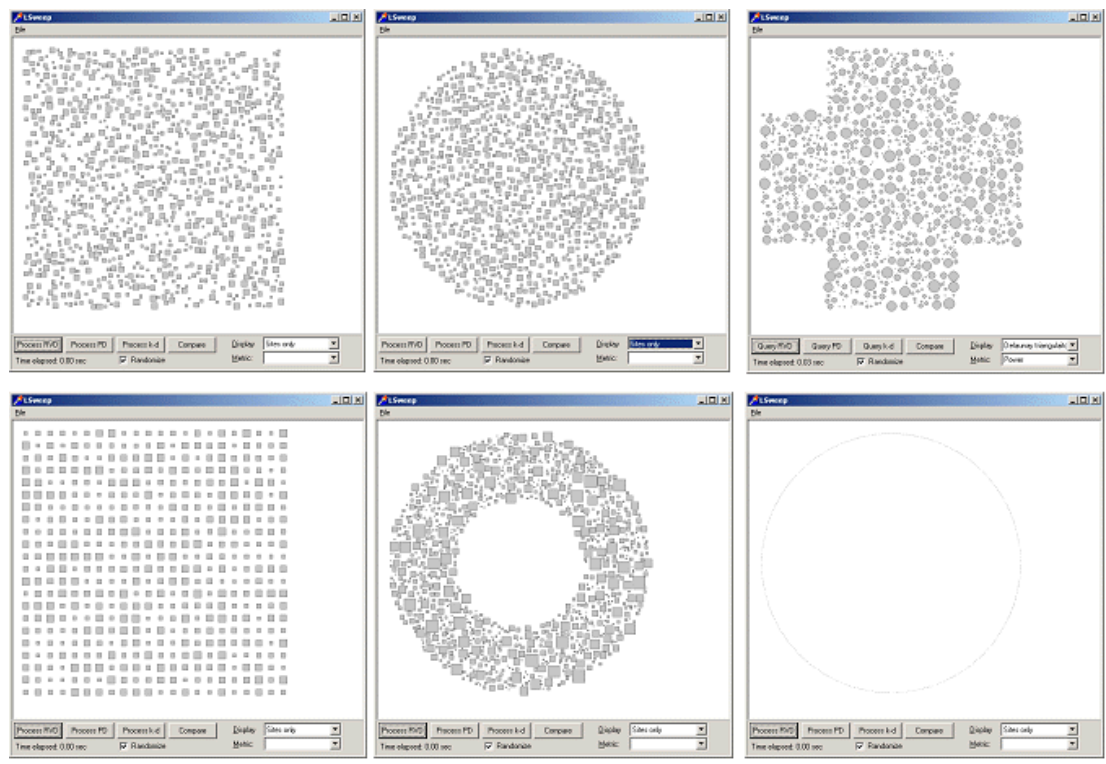

Fig. 2. Six configurations of sites in supremum metric (left right, top down direction): uniform square, uniform circle, cross, degenerate grid, ring and degenerate circle.

specified input configuration. Then NNM performs a series of nearest-neighbor searches.

The efficiency of the VD-based method was compared against the $k-d$ tree method for a set of circles in the plane. The $k-d$ tree implementation is based on the Ranger software [13], which implements an optimal $k-d$ tree method for a set of points [6]. The software was modified to accommodate the circular sites. Each site was represented by the four corner points, effectively allowing reporting the nearest neighbor in the weighted supremum metric. The software was also optimized to avoid the unnecessary memory allocations and initializations of the internal variables for maximum efficiency when performing multiple queries. The efficiency of the method does not depend on the metric used, thus the supremum and power VD methods were compared to the same implementation of the $k-d$ tree method.

After the initial distribution is generated, it is loaded into the NNM Module. First, the generalized Voronoi diagram, Delaunay Triangulation or a $k-d$ tree is computed in power or supremum metric. The snapshot of the screen (see Fig. 3) illustrates the Voronoi diagram in Laguerre geometry of 10000 circular sites in ring configuration. The nearest-neighbor queries are done by either generating a sequence of random queries or by selecting the query point manually. When a manual query is performed, the path from the starting VD edge to the nearestneighbor of the query point is highlighted and the length of this path is displayed. 


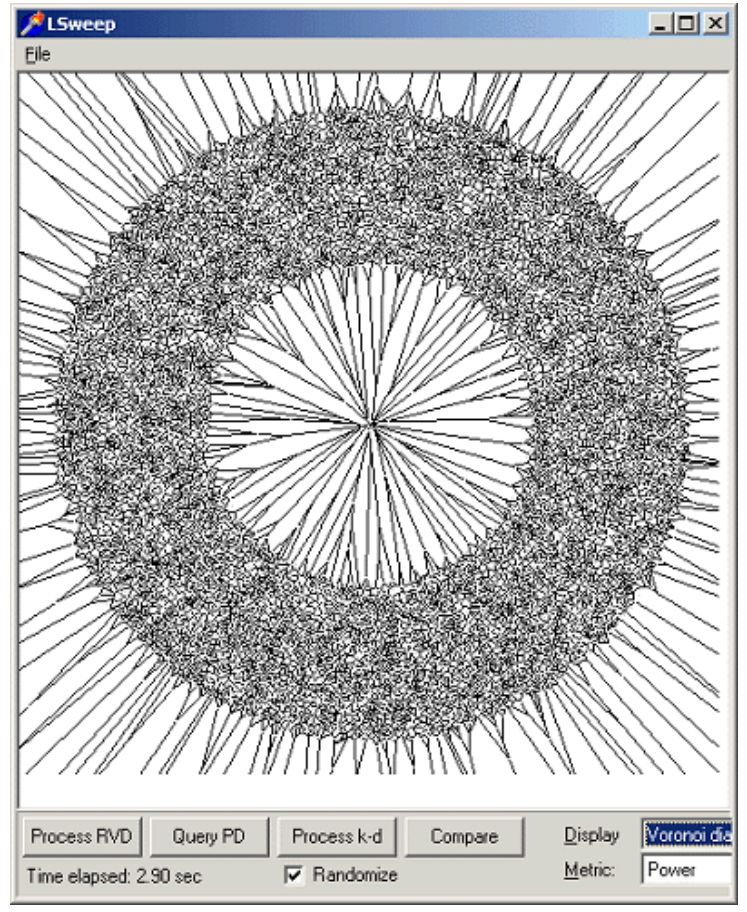

Fig. 3. Example of generalized VD under power metric for 10000 sites, ring configuration

The measured characteristics that represent the performance of the method include the total number of queries performed, the elapsed time, the average time per query, and the average search length. The average search length is a parameter related to the number of comparisons that the algorithm performs. For the VD approach, this parameter represents the total number of edges that were encountered during the edge walk while performing a query. In case of $k-d$ tree this parameter represents the total number of distance comparisons performed on different nodes of the tree. This parameter was selected for evaluation since it helps to compare VD and $k-d$ tree methods, and it can be easily visualized.

\section{$5 \quad$ Experimental Studies}

The experiments were performed for different data set sizes, various distributions of their density, radii and site configurations. The algorithms were tested on the generated data sets and the data set representing granular-type material system for a silos simulation with large number of particles (the data sets were provided by the Department of Mechanical Engineering, University of Calgary). 
Uniform Square: Initialization Time vs. Sites

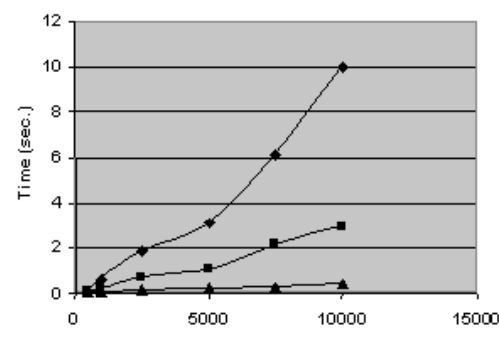

Number of sites
Degenerate Grid: Initialization Time vs. Sites

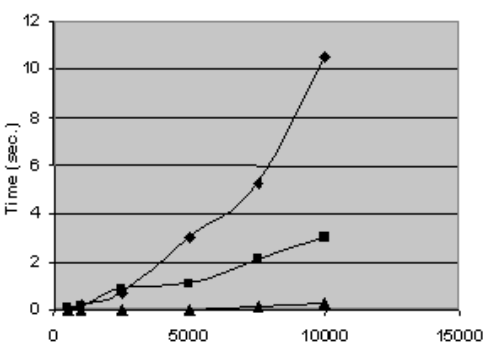

Number of sites

Degenerate Circle Initialization Time vs. Sites

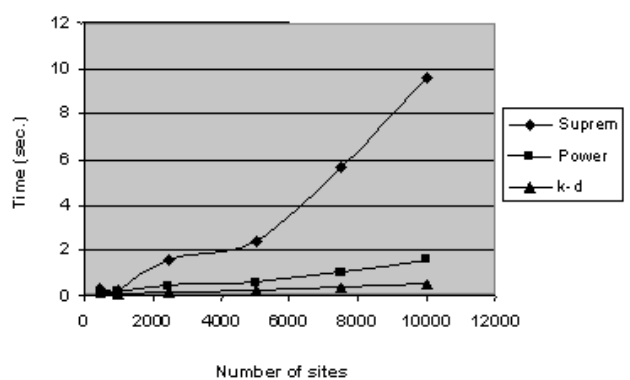

Fig. 4. Time required for building the initial data structure vs. the number of sites

The first series of experiments were performed on randomly generated distributions including uniform square, uniform circle, cross, ring, degenerate grid and degenerate circle distributions. All of the distributions were tested on data sets consisting of 100 to 10000 input sites. The experiments show that the $k-d$ tree method requires much less initialization time than the Voronoi diagram methods, even though the upper bound for both algorithms is $O(n)$. Experimental results demonstrated that the initialization time required to build the data structure is the smallest for the $k-d$ tree based method (see Fig. 41). However, both the power diagram and supremum diagram method consistently outperformed the $k-d$ tree method in terms of the query time required to find the nearest-neighbor (see Fig. 5). This holds for regular as well as close to degenerate configurations. Note that the query time for both VD based methods is very close. The average search length was recorded for all the tests performed, and it exposed a similar linear dependence (growth) as the number of sites increased. Thus, for uniform and degenerate grid distributions it increases from 10 for 100 sites to about 180 for 10000 sites. In the case of circle distribution it increases from 50[DG1] for 100 sites to 5500 for 10000 sites. This result is consistent with the fact that the queries on the circle distribution are usually more time consuming than the queries performed on all other distributions. Based on the results obtained, the 
Uniform Square: Query Time vs. Sites

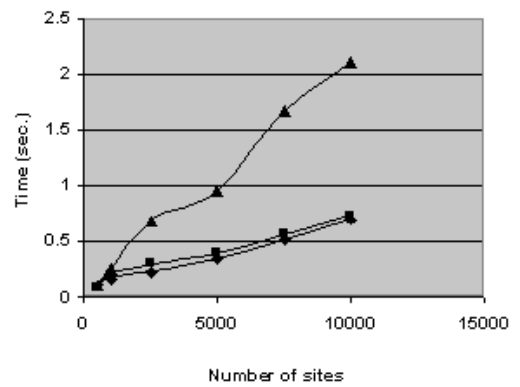

Degenerate Grid: Query Time vs. Sites

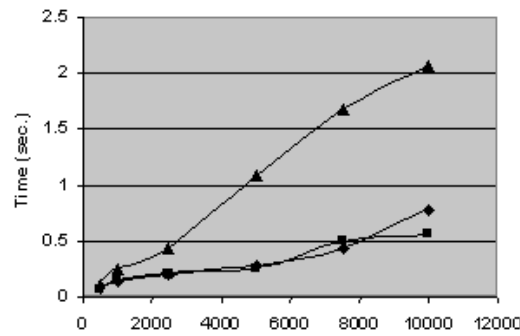

Number of sites

Degenerate Circle: Query Time vs. Sites

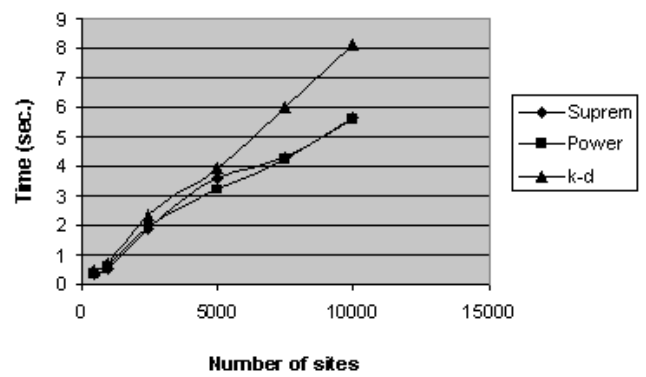

Fig. 5. Time required for performing 1000 queries vs. the number of sites

conclusion can be made that the power and supremum Voronoi diagram method is an efficient data structure for performing nearest-neighbor queries, independent of the site configurations. This was demonstrated for the number of input sites increasing from 100 to 10000 . However, the preprocessing time for the VDbased method is quite large compared to the $k-d$ tree method.

Another interesting result is that the VD in either metric can be used for approximate nearest-neighbor searches. The following series of experiments were performed to determine how 'close' the nearest-neighbor found in power metric would be to the nearest-neighbor reported in the supremum metric . The experimental results show that in $95 \%$ of all cases the same nearest-neighbor is reported in both metrics, and in $4.5 \%$ of remaining cases the two nearestneighbors reported in different metrics were connected by an edge in the Delaunay triangulation. This shows that it is possible to use either a power diagram or a supremum Voronoi diagram for the approximate nearest-neighbor searches. The third series of the experiments were performed on a data set generated as a result of computer simulation of the granular-type material system for a silo model [7]. The model represents a grain elevator with vertical boundaries and a large number of densely packed grain particles. Test results show that the initialization time for the power diagram method is practically the same as for the 


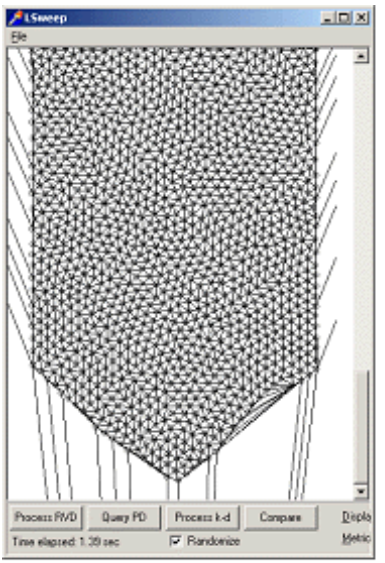

\section{Silo: Query time vs. Number of Sites (1000 queries)}

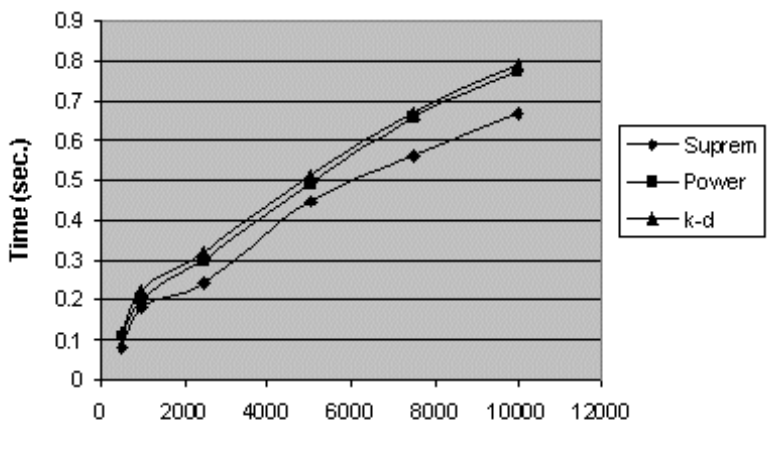

Number of sites

Fig. 6. DT built in power metric for 2500 particles and the running time vs. number of sites.

$k-d$ tree method and the supremum diagram requires significantly more time for initialization. The query time for power diagram is almost the same as for supremum diagram and outperforms the $k-d$ tree method (see Fig. 6).

\section{Conclusions}

This paper presented an algorithm for an efficient solution of the nearestneighbor problem for a set of weighted sites based on the generalized Delaunay triangulation. The results obtained clearly demonstrate the applicability of the generalized DT under various distance functions as an efficient, robust and easy to implement method for solving the nearest-neighbor problem. The investigation of the different approaches to select the starting site for the search represents an interesting problem.

Author would like to thank Jon Rokne and Nikolai Medvedev for useful comments and suggestions that helped to improve the paper. Author would also like to express special thanks to Dmitri Gavrilov and Oleg Vinogradov for providing the test data. The work was partly supported by UCRG Research Grant.

\section{References}

[1] Aggarwal, P., Raghawan, P. Deferred data structures for the nearest-neighbor problem, Inform. Process. Letters 40 (3) (1991) 119-122.

[2] Bentley J. L. $k-d$ Trees for Semidynamic Point Sets, in Proceedings of the 6th Annual ACM Symposium on Computational Geometry (1990) 187-197. 
[3] Berchtold, S., Ertl, B., Keirn, D., Kriegel, H.P., Seidel, T. Fast nearest neighbor search in high-dimensional space, in Proc. of the 14th Intn. Conf. On Data Emg, Orlando, Florida (1998).

[4] Devillers, O., Golin, M., Kedem, K., Schirra, S. Queries on Voronoi Diagrams of Moving Points, Comput. Geom. Theory and Applic. 6 (1996) 315-327.

[5] Edelsbrunner, H., Shah, N. Incremental topological flipping works for regular triangulations, Algorithmica 15 (1996) 223-241.

[6] Friedman, J., Bentley, J., Finkel, R. An Algorithm for Finding Best Matches in Logarithmic Expected Time, ACM Transactions on Mathematical Software, 3(3) (1977) 209-226.

[7] Gavrilov, D., Vinogradov, O. A cluster in granular systems as a topologically variable structure, in Proc. of 1997 ASCE Symposium on Mechanics of Deformation and Flow of Particulate Materials, Evanston, IL (1997) 299-307.

[8] Gavrilova, M. Proximity and Applications in General Metrics Ph. D. Thesis, Dept. of Computer Science, University of Calgary, Canada (1999).

[9] Gavrilova, M., Rokne, J., Vinogradov O and Gavrilov D. Collision detection algorithms in simulation of granular materials, 1999 ASME Mechanics and Materials Conference, (1999) 283- 284.

[10] Graf, T., Hinrichs, K. A Plane-Sweep Algorithm for the All-Nearest-Neighbors Problem for a Set of Convex Planar Objects, in Proc. 3rd Works. Algm. Data Struct., LNCS, Springer-Verlag 709 (1993) 349-360.

[11] Luchnikov, V.A., Medvedev, N.N., Voloshin, V.P., Geiger, A. Simulation of transport and diffusion of the Voronoi network, in the book: Scientific Computing in Chemical Engineering, Springer-Verlag, Berlin, (1999).

[12] Medvedev, N.N. Voronoi-Delaunay Method for Non-crystalline Structures, SB Russian academy of Science, Novosibirsk (in Russian) (2000).

[13] Murphy, M., Skiena, S. A study of data structures for orthogonal range and nearest neighbor queries in high dimensional spaces, CSE 523/524 Master's Project, Department of Computer Science, SUNYSB (1996).

[14] Okabe, A., Boots, B., Sugihara, K. Spatial Tessellations: Concepts and Applications of Voronoi Diagrams. John Wiley \& Sons, Chichester, England (1992).

[15] O'Rourke, J. Computational geometry in C. Cambridge Univ. Press (1994)

[16] Schaudt, B., Drysdale, R. Higher-dimensional Voronoi diagrams for convex distance functions," in Proc. of the 4th Can. Conf. on Comp. Geometry (1992) 274-279.

[17] Shinoda, W., Okazaki, S. A Voronoi analysis of lipid area fluctuation in a bilayer J. Chem. Phys. V. 109(4) (1998) 1517-1521. 